\title{
Pacific
}

Journal of

Mathematics

\section{ON A THEOREM OF PAUL YANG ON NEGATIVELY PINCHED} BISECTIONAL CURVATURE

\author{
AERYEONG SEO
}




\title{
ON A THEOREM OF PAUL YANG ON NEGATIVELY PINCHED BISECTIONAL CURVATURE
}

\author{
AERYEONG SEO \\ We present examples of domains that do not admit any complete Kähler \\ metric with bisectional curvature bounded between prescribed two negative \\ constants by a modification of a method of $P$. Yang.
}

\section{Introduction}

Yang [1976] showed that the polydiscs of complex dimension at least two do not admit any complete Kähler metrics with their holomorphic bisectional curvature bounded between two negative constants. He also pointed out that the same argument applies to the bounded symmetric domains with rank higher than one; these domains therefore do not admit such metrics with the same curvature condition. On the other hand the Poincaré-Bergman metric of the unit ball is a complete Kähler metric with its bisectional curvature bounded between two negative constants. Thus the following question [Yau 1982] seems natural:

Which complex manifolds admit a complete Kähler metric with bisectional curvature bounded between two negative constants?

Yang's original interest was linked with a question on the holomorphic universal covering manifold of a compact Kähler manifold with negative curvature, it was conjectured that it should be biholomorphic to a bounded domain. Since such a universal cover was shown to be a Stein manifold by Greene and Wu [1971;1979] during that period, Yang's work was a natural one in the sense that he investigated the curvature of possible Kähler metrics on bounded symmetric domains. On the other hand, the question posed above seems, in its own right, to contain sufficient significance to deserve further study. Incidentally, the method developed in [Yang 1976], since it is the almost unique one known to this day, also seems worth deeper investigation.

The author was supported in part by the grant number 4.0006570 (2011, PI: Kang-Tae Kim) of The Basic Science Research Institute of Pohang University of Science and Technology, The Republic of Korea.

MSC2010: primary 32Q05; secondary 32Q15.

Keywords: bisectional curvature, negative curvature, complete Kähler metrics. 
Indeed, Seshadri and Zheng [2008] investigated this line of thought and showed that Yang's method works not only for domains but also for product manifolds. It occurred to us that it is meaningful to investigate whether there are manifolds which do not admit complete Kähler metrics with negative bisectional curvature that are neither biholomorphic to product nor homogeneous manifolds. In fact the main purpose of this paper is to present a modification of Yang's method and applications, and the following theorems:

Theorem 1.1. There exists a domain $\Omega$ in $\mathbb{C}^{2}$ satisfying the following properties:

(i) $\Omega$ is a bounded pseudoconvex domain with smooth boundary.

(ii) Aut $\Omega$ does not act transitively on $\Omega$.

(iii) $\Omega$ is not biholomorphic to the product of complex manifolds.

(iv) $\Omega$ does not admit complete Kähler metric with bisectional curvature bounded between two negative constants.

Theorem 1.2. Let $\Omega=\left\{(z, w) \in \mathbb{C}^{2}:|z|<1,|w|<r(z)\right\}$, where $r: D \rightarrow \mathbb{R}$ is a smooth positive function on $D$ such that $\frac{\partial^{2}}{\partial z \partial \bar{z}} \frac{1}{r(z)^{2}}<0$. Then $\Omega$ cannot admit any complete Kähler metric with bisectional curvature bounded between two negative constants.

The rest of the paper is organized as follows: Section 2 introduces some basic facts and terminology. In Section 3, our modification of Yang's proof is presented with a proof of Theorem 1.1. In Section 4, we give yet another modification of Yang's method and the proof of Theorem 1.2.

\section{Preliminary and fundamental facts}

First, we introduce some facts and terminology. Let $(M, J, h)$ be a Kähler manifold $M$ of dimension $n$ with a Kähler metric $h$ and a complex structure $J$. The curvature tensor $R$ on $(M, J, h)$ is given by

$$
R_{i \bar{j} k \bar{l}}=\frac{\partial^{2} h_{i \bar{j}}}{\partial z_{k} \partial \bar{z}_{l}}-\sum_{\alpha, \beta=1}^{n} h^{\alpha \bar{\beta}} \frac{\partial h_{i \bar{\beta}}}{\partial z_{k}} \frac{\partial h_{\alpha \bar{j}}}{\partial \bar{z}_{l}}
$$

in local coordinates $\left(z_{1}, \ldots, z_{n}\right)$. The bisectional curvature $B(X, Y)$ for $X, Y$ in $T_{p} M$ at $p \in M$ is given by

$$
B(X, Y)=\frac{R(X, J X, Y, J Y)}{h(X, X) h(Y, Y)} .
$$

In terms of local coordinates,

$$
B(X, Y)=-\frac{\sum_{i, j, k, l=1}^{n} R_{i \bar{j} k \bar{l}} X_{i} \bar{X}_{j} Y_{k} \bar{Y}_{l}}{\sum_{i, j=1}^{n} h_{i j} X_{i} \bar{X}_{j} \sum_{i, j=1}^{n} h_{i j} Y_{i} \bar{Y}_{j}}
$$


where

$$
X=\operatorname{Re} \sum_{j=1}^{n} X_{j} \frac{\partial}{\partial z_{j}} \quad \text { and } \quad Y=\operatorname{Re} \sum_{j=1}^{n} Y_{j} \frac{\partial}{\partial z_{j}} .
$$

The following well known theorems will play important roles in our exposition: Theorem 2.1 (generalization of Schwarz's lemma [Yau 1978]). Let $(M, g)$ be a complete Kähler manifold with Ricci curvature bounded below by a constant $-k$ and let $(N, h)$ be a Hermitian manifold with bisectional curvature bounded above by a negative constant $-K$. Then every holomorphic mapping $f: M \rightarrow N$ satisfies

$$
f^{*} h \leq \frac{k}{K} g .
$$

Theorem 2.2 (generalized maximum principle [Omori 1967; Yau 1975]). Let M be a complete Riemannian manifold with Ricci curvature bounded below. Then for every $C^{2}$ function $f: M \rightarrow \mathbb{R}$ that is bounded from above, there exists a sequence $\left\{p_{k}\right\}_{k=0}^{\infty}$ in $M$ such that

$$
\lim _{k \rightarrow \infty}\left|\operatorname{grad} f\left(p_{k}\right)\right|=0, \quad \limsup _{k \rightarrow \infty} \Delta f\left(p_{k}\right) \leq 0 \quad \text { and } \quad \lim _{k \rightarrow \infty} f\left(p_{k}\right)=\sup _{M} f .
$$

For $D_{r}=\{z \in \mathbb{C}:|z|<r\}$, denote by

$$
g_{r}=\frac{r^{2} d z d \bar{z}}{\left(r^{2}-|z|^{2}\right)^{2}}
$$

the Poincare metric on $D_{r}$ with curvature -4 . Let $D$ be the unit disc and $g$ the Poincaré metric on $D$. Denote by $D^{n}$ the $n$-dimensional polydisc. Let $\operatorname{grad}_{h}$ and $\triangle_{h}$ be the gradient and the Laplacian with respect to the Riemannian metric $h$.

\section{A modified proof of the theorem of Paul Yang}

We begin this section by proving Yang's theorem again, using a modification of his method; our modification lies in that we do not use integrals.

Theorem 3.1 [Yang 1976]. The polydisc $D^{n}(n \geq 2)$ and bounded symmetric domains with rank $\geq 2$ do not admit any complete Kähler metrics with its bisectional curvature bounded between two negative constants.

Proof. For simplicity, we will prove the result for $D^{2}$. However, the same proof can be applied to higher-dimensional polydiscs and bounded symmetric domains with rank $\geq 2$. Suppose that there exists a complete Kähler metric $h$ on $D^{2}$ with $-d \leq B(X, Y) \leq-c<0$ for any $X, Y$, where $c, d$ are positive constants. Fix $z \in D$ and define $i_{z}: D \rightarrow D^{2}$ by $i_{z}(w)=(z, w)$. By Theorem 2.1, we have $i_{z}^{*} h \leq \frac{4}{c} g$; that is,

$$
h_{2 \overline{2}}(z, w) \leq \frac{4}{c} \frac{1}{\left(1-|w|^{2}\right)^{2}} .
$$


Define $F: D \rightarrow \mathbb{R}$ by $F(z)=h_{2 \overline{2}}(z, 0)$. Then $F$ is a smooth, positive, bounded function. We will induce a contradiction by calculating the Laplacian of $F$ :

$$
\begin{aligned}
\triangle_{g} F(z) & =\left(1-|z|^{2}\right)^{2} \frac{\partial^{2} F}{\partial z \partial \bar{z}}(z) \\
& =\left(1-|z|^{2}\right)^{2}\left(R_{1 \overline{1}} \overline{2}(z, 0)+\sum_{\alpha, \beta=1}^{2} h^{\alpha \bar{\beta}} \frac{\partial h_{2 \bar{\beta}}}{\partial z} \frac{\partial h_{\alpha \overline{2}}}{\partial \bar{z}}\right) \\
& \geq c\left(1-|z|^{2}\right)^{2} h_{2 \overline{2}}(z, 0) h_{1 \overline{1}}(z, 0) .
\end{aligned}
$$

From Schwarz's lemma applied to $\pi: D^{2} \rightarrow D, \pi(z, w)=z$, we get $\pi^{*} g \leq \frac{2 d}{4} h$, that is, $\left(1-|z|^{2}\right)^{2} h_{1 \overline{1}}(z, w) \geq 2 / d$. So

$$
\triangle_{g} F \geq \frac{2 c}{d} F
$$

Since $\triangle_{g} \log F=\triangle_{g} F /|F|-\left|\operatorname{grad}_{g} F\right|^{2} /|F|^{2}$ and $\log F$ is a well defined bounded function, by the almost maximum principle (Theorem 2.2) there exists a sequence $\left\{p_{k}\right\}_{n=1}^{\infty}$ in $D$ such that

$\lim _{k \rightarrow \infty} F\left(p_{k}\right)=\sup _{D} F, \quad \lim _{k \rightarrow \infty}\left|\operatorname{grad}_{g} \log F\left(p_{k}\right)\right|=0 \quad$ and $\quad \limsup _{k \rightarrow \infty} \triangle_{g} \log F\left(p_{k}\right) \leq 0$.

But this contradicts $\lim \sup _{k \rightarrow \infty} \triangle_{g} \log F\left(p_{k}\right) \geq 2 c / d$.

Though clear, the crux of the above argument can be summarized as follows:

Proposition 3.2. Let $\Omega$ be a domain in $\mathbb{C}^{n}$. Suppose that there exist an embedding

$$
\iota: D^{2} \rightarrow \Omega, \quad \iota(z, w)=(z, w, 0, \ldots, 0)
$$

and a projection

$$
\pi: \Omega \rightarrow D, \quad \pi\left(z_{1}, z_{2}, \ldots, z_{n}\right)=z_{1} .
$$

Then for any constants $c>d>0$, there is no complete Kähler metric on $\Omega$ with

$$
-c \leq \text { Bisec } \leq-d .
$$

Example 3.3. Let $\Omega=\left\{(z, w, u) \in \mathbb{C}^{2}:|z|<1,|w|<\alpha,|z+w|<\beta\right\}$. If $\alpha \geq 1$ and $\beta \geq 2$, then $\Omega$ does not have any complete Kähler metrics with bisectional curvature bounded between two negative constants. This $\Omega$ is a nonproduct and nonhomogeneous analytic polyhedron that is not biholomorphic to the bidisc: If $\Omega$ were a product manifold, $\Omega$ would be biholomorphic to $D^{2}$ by the Riemann mapping theorem. However, $\Omega$ cannot be biholomorphic to $D^{2}$ [Fridman 1978]. Likewise, $\Omega$ is not homogeneous; if it were, then it would be biholomorphic to $D^{2}$ [Kim 1992]. 
For a sequence of domains $\left\{\Omega_{p}\right\}_{p=1}^{\infty}$, it is possible that $\Omega_{p}$ does not admit any complete Kähler metrics with bisectional curvature bounded between two negative constants for all $p$, but that $\left\{\Omega_{p}\right\}$ converges to the ball as $p \rightarrow \infty$.

Corollary 3.4. For any $0<r<1$ and positive constants $0<c<d$,

$$
\Omega=\left\{(z, w) \in \mathbb{C} \times \mathbb{C}^{n}:|z|^{2}+|w|^{2}<1,|z|<r\right\}
$$

does not admit complete Kähler metric with

$$
-d<\text { Bisec }<-c \text {. }
$$

Proof. The mapping

$$
(z, w) \mapsto\left(\frac{1}{r} z, \frac{1}{\sqrt{1-r^{2}}} w\right)
$$

sends $\Omega$ to $\left\{(z, w) \in \mathbb{C} \times \mathbb{C}^{n}: r^{2}|z|^{2}+\left(1-r^{2}\right)|w|^{2}<1,|z|<1\right\}$. So an embedding and a projection like in Proposition 3.2 exist.

Remark 3.5. In contrast, the sequence of domains $\left\{\Omega_{m}\right\}_{m=1}^{\infty}$, where the domain $\Omega_{m}=\left\{(z, w) \in \mathbb{C}^{n}:|z|^{2}+|w|^{2 m}<1\right\}$ has a complete Kähler metric with sectional curvature bounded between two negative constants for all $m$, converges to the bidisc. Bland [1986] proved that the Kähler-Einstein metric on $\Omega_{m}$ has sectional curvature bounded between two negative constants. However we could find another complete Kähler metric explicitly which has sectional curvature bounded between two negative constants.

On $\Omega_{m}=\left\{(z, w) \in \mathbb{C}^{n}:|z|^{2}+|w|^{2 m}<1\right\}$, let

$$
g_{m}(z, w)=-\partial \bar{\partial} \log \rho(z, w), \quad \text { where } \rho(z, w)=\left(1-|z|^{2}\right)^{\frac{1}{m}}-|w|^{2} .
$$

Then $g_{m}$ is a complete Kähler metric on $\Omega_{m}$. The metric $g_{m}(z, w)$ is given by

$$
\frac{\frac{1}{m}\left(1-|z|^{2}\right)^{\frac{1}{m}-2}}{\left(\left(1-|z|^{2}\right)^{\frac{1}{m}}-|w|^{2}\right)^{2}}\left(\begin{array}{cc}
\left(1-|z|^{2}\right)^{\frac{1}{m}}-|w|^{2}+\frac{1}{m}|z|^{2}|w|^{2} & w \bar{z}\left(1-|z|^{2}\right) \\
\bar{w} z\left(1-|z|^{2}\right) & m\left(1-|z|^{2}\right)^{2}
\end{array}\right),
$$

where

$$
\operatorname{det} g_{m}=\frac{\frac{1}{m}\left(1-|z|^{2}\right)^{\frac{2}{m}-2}}{\left(\left(1-|z|^{2}\right)^{\frac{1}{m}}-|w|^{2}\right)^{3}}
$$

and the inverse $g_{m}^{-1}(z, w)$ is

$$
\frac{\left(1-|z|^{2}\right)^{\frac{1}{m}}-|w|^{2}}{\left(1-|z|^{2}\right)^{\frac{1}{m}}}\left(\begin{array}{cc}
m\left(1-|z|^{2}\right)^{2} & -w \bar{z}\left(1-|z|^{2}\right) \\
-\bar{w} z\left(1-|z|^{2}\right) & \left(1-|z|^{2}\right)^{\frac{1}{m}}-|w|^{2}+\frac{1}{m}|z|^{2}|w|^{2}
\end{array}\right) .
$$


This metric $g_{m}$ is invariant with respect to the automorphisms

$$
f_{\alpha}(z, w)=\left(\frac{\alpha-z}{1-\bar{\alpha} z}, \frac{\left(1-\alpha^{2}\right)^{\frac{1}{2 m}}}{(1-\bar{\alpha} z)^{\frac{1}{m}}} w\right) \quad \text { for } \alpha \in D,
$$

so it is enough to calculate the section curvature at $(0, w)$. By calculation,

$$
\begin{aligned}
2 m g_{1 \overline{1}}^{2}> & R_{1 \overline{1} 1 \overline{1}}=2 g_{1 \overline{1}}^{2}\left(|w|^{2}+m\left(1-|w|^{2}\right)\right)>2 g_{1 \overline{1}}^{2}, \\
R_{2 \overline{2} 2 \overline{2}} & =2 g_{2 \overline{2}}^{2}, \\
R_{1 \overline{1} 2 \overline{2}} & =R_{2 \overline{2} 1 \overline{1}}=R_{1 \overline{2} 2 \overline{1}}=R_{2 \overline{1} 1 \overline{2}}=g_{1 \overline{1}} g_{2 \overline{2}},
\end{aligned}
$$

and the other $R_{i \bar{j} k \bar{l}}$ are zero at $(0, w)$. This implies that

$$
-2 m<\text { sectional curvature of } \Omega_{m}<-\frac{1}{2} .
$$

However the lower bound on the sectional curvature of this metric and of the Kähler-Einstein metric depends on $m$ and goes to $-\infty$ as $m \rightarrow \infty$. So this question is still open:

If $\Omega_{m}$ has a complete Kähler metric with $-d<($ bi)sect $<-c$ for some constants $c, d>0$ for all $m$ and converges to $\Omega$ as $m \rightarrow \infty$, then does $\Omega$ have a complete Kähler metric with $-d<($ bi)sect $<-c$ ?

Proof of Theorem 1.1. For $\epsilon>0, \alpha<1$, let

$$
\begin{aligned}
& \Omega_{\epsilon}=\left\{(z, w) \in \mathbb{C}^{2}:|z|^{2}+\alpha e^{-1 /\left(|w|^{2}-\epsilon\right)}<1,|w|>1 / \sqrt{\epsilon}\right\} \\
& \cup\left\{(z, w) \in \mathbb{C}^{2}:|z|<1,|w| \leq 1 / \sqrt{\epsilon}\right\}
\end{aligned}
$$

Then $\Omega_{\epsilon}$ is a nonhomogeneous, nonproduct, bounded pseudoconvex domain with smooth boundary. The points $(z, w) \in \partial \Omega$ with $|w|^{2}>\epsilon$ are strongly pseudoconvex. By dilating $(z, w) \mapsto(z, w / \sqrt{\epsilon})$, we see that $\Omega_{\epsilon}$ is biholomorphic to

$$
\left\{(z, w) \in \mathbb{C}^{2}:|z|^{2}+\alpha^{2} \exp \left(1-1 /\left(\epsilon|w|^{2}-\epsilon\right)\right)<1,|z|<1\right\} \cup D^{2} .
$$

Therefore we can apply Proposition 3.2.

\section{Further modification}

We now find more examples of domain that do not admit complete Kähler metric with bisectional curvature bounded between two negative constants. The domains we look for will be different from analytic polyhedra. We will achieve this by modifying Yang's method one more time.

Proof of Theorem 1.2. Suppose that there exists a complete Kähler metric $h$ on $\Omega$ with $-d \leq B(X, Y) \leq-c<0$ for any $X, Y$ for some positive constants $c, d$. 
Define $i_{z}: D_{r(z)} \rightarrow \Omega$ by $i_{z}(w)=(z, w)$. By Schwarz's lemma, $i_{z}^{*} h \leq \frac{4}{c} g_{r(z)}$. So $h_{2 \overline{2}}(z, w) \leq \frac{4}{c} r(z)^{2} /\left(r(z)^{2}-|w|^{2}\right)^{2}$. Define $F: D \rightarrow \mathbb{R}$ by

$$
F(z)=r(z)^{2} h_{2 \overline{2}}(z, 0) .
$$

Then $F$ is a smooth, positive, bounded function. We have

$$
\frac{\partial F}{\partial z}(z)=\frac{\partial r^{2}}{\partial z}(z) h_{2 \overline{2}}(z, 0)+r(z)^{2} \frac{\partial h_{2 \overline{2}}}{\partial z}(z, 0)
$$

and

$$
\begin{aligned}
\frac{\partial^{2} F}{\partial z \partial \bar{z}} & =r^{2} \frac{\partial^{2} h_{2 \overline{2}}}{\partial z \partial \bar{z}}+\frac{\partial^{2} r^{2}}{\partial z \partial \bar{z}} h_{2 \overline{2}}+2 \operatorname{Re}\left(\frac{\partial r^{2}}{\partial z} \frac{\partial h_{2 \overline{2}}}{\partial \bar{z}}\right) \\
& =r^{2} \frac{\partial^{2} h_{2 \overline{2}}}{\partial z \partial \bar{z}}+\frac{\partial^{2} r^{2}}{\partial z \partial \bar{z}} h_{2 \overline{2}}+2 \operatorname{Re}\left(\frac{\partial r^{2}}{\partial z} \frac{1}{r^{2}}\left(\frac{\partial F}{\partial \bar{z}}-\frac{\partial r^{2}}{\partial \bar{z}} h_{2 \overline{2}}\right)\right) \\
& =r^{2} \frac{\partial^{2} h_{2 \overline{2}}}{\partial z \partial \bar{z}}+\left(\frac{\partial^{2} r^{2}}{\partial z \partial \bar{z}}-\frac{2}{r^{2}}\left|\frac{\partial r^{2}}{\partial z}\right|^{2}\right) h_{2 \overline{2}}+\frac{2}{r^{2}} \operatorname{Re}\left(\frac{\partial r^{2}}{\partial z} \frac{\partial F}{\partial \bar{z}}\right) \\
& =r^{2} \frac{\partial^{2} h_{2 \overline{2}}}{\partial z \partial \bar{z}}-r^{4} \frac{\partial^{2} r^{-2}}{\partial z \partial \bar{z}} h_{2 \overline{2}}+\frac{2}{r^{2}} \operatorname{Re}\left(\frac{\partial r^{2}}{\partial z} \frac{\partial F}{\partial \bar{z}}\right) .
\end{aligned}
$$

Thus

$$
\begin{aligned}
\triangle_{g} F & \geq c\left(1-|z|^{2}\right)^{2} r^{2} h_{2 \overline{2}} h_{1 \overline{1}}-\left(1-|z|^{2}\right)^{2} r^{2} \frac{\partial^{2} r^{-2}}{\partial z \partial \bar{z}} F+\frac{2\left(1-|z|^{2}\right)^{2}}{r^{2}} \operatorname{Re}\left(\frac{\partial r^{2}}{\partial z} \frac{\partial F}{\partial \bar{z}}\right) \\
& \geq \frac{2 c}{d} F-\left(1-|z|^{2}\right)^{2} r^{2} \frac{\partial^{2} r^{-2}}{\partial z \partial \bar{z}} F+\frac{2\left(1-|z|^{2}\right)^{2}}{r^{2}} \operatorname{Re}\left(\frac{\partial r^{2}}{\partial z} \frac{\partial F}{\partial \bar{z}}\right) .
\end{aligned}
$$

So if $r$ satisfies $\frac{\partial^{2} r^{-2}}{\partial z \partial \bar{z}}<0$, then the desired result follows by the almost maximum principle as in the previous modification of Yang's proof.

Corollary 4.1. Let $\Omega$ is the domain in the Theorem 1.2. Suppose $1 / r(z)^{2}=\rho(|z|)$ for some positive function $\rho$ on $[0,1]$ and suppose

$$
t \frac{d \rho}{d t}(t)
$$

is a decreasing function. Then $\Omega$ does not admit any complete Kähler metrics with bisectional curvature bounded between any two prescribed negative constants.

Proof. This follows by

$$
\frac{\partial^{2} r^{-2}}{\partial z \partial \bar{z}}=\frac{d^{2} \rho}{d t^{2}}+\frac{1}{t} \frac{d \rho}{d t}=\frac{1}{t}\left(\frac{d}{d t}\left(t \frac{d \rho}{d t}\right)\right) .
$$

Typical examples of $\rho$ and $\Omega$ satisfying the conditions in Corollary 4.1 are:

Example 4.2. Let $\rho(t)=\exp (-t)$ and

$$
\Omega=\left\{(z, w) \in \mathbb{C}^{2}:|z|<1,|w|^{2}<\exp (|z|)\right\} .
$$


Example 4.3. Let $\rho(t)=\alpha-t^{2}$ and

$$
\Omega=\left\{(z, w) \in \mathbb{C}^{2}:|z|<1,|w|^{2}\left(\alpha-|z|^{2}\right)<1\right\} .
$$

If we take $\alpha>1$, then $\Omega$ is a bounded domain.

Remark 4.4. If

$$
\frac{\partial^{2} r^{-2}}{\partial z \partial \bar{z}}<0 \quad \text { then } \quad \frac{\partial^{2} r^{2}}{\partial z \partial \bar{z}}>\frac{2}{r^{2}}\left|\frac{\partial r^{2}}{\partial z}\right|^{2}
$$

Notice that the domains given in Theorem 1.2 are in fact pseudoconcave.

The domains in Theorem 1.2 converge to $\left\{(z, w) \in \mathbb{C}^{2}:|z|^{2}+\alpha \exp \left(\frac{-1}{|w|^{2}}\right)<1\right\}$ as $\epsilon \rightarrow 0$, which does not admit a complete Kähler metric with $-d<$ Bisec $<-c$ for some constants $c, d>0$ :

Corollary 4.5. Let $\Omega=\left\{(z, w) \in \mathbb{C}^{2}:|z|^{2}+\alpha \exp \left(\frac{-1}{|w|^{2}}\right)<1\right\}$, for some constant $\alpha>1$. For any positive constants $c<d$, such that $c / d>(2 \log \alpha)^{-1}, \Omega$ cannot admit a complete Kähler metric with

$$
-d \leq \operatorname{Bisec} \leq-c .
$$

Proof. Notice that

$$
\Omega=\left\{(z, w) \in \mathbb{C}^{2}:|z|<1,|w|<r(z)\right\}
$$

where $r(z)=1 /\left(\log \alpha-\log \left(1-|z|^{2}\right)\right)^{\frac{1}{2}}$. Since $\frac{\partial^{2} r^{-2}}{\partial z \partial \bar{z}}=\frac{1}{\left(1-|z|^{2}\right)^{2}}$, we obtain for

$$
F: D \rightarrow \mathbb{R}, \quad F(z)=r(z)^{2} h_{2 \overline{2}}(z, 0)
$$

the inequality

$$
\triangle_{g} F(z) \geq \frac{2 c}{d} F(z)-\frac{F}{\log \alpha-\log \left(1-|z|^{2}\right)}-\frac{2\left(1-|z|^{2}\right)}{\log \alpha-\log \left(1-|z|^{2}\right)} \operatorname{Re}\left(\bar{z} \frac{\partial F}{\partial \bar{z}}(z)\right) .
$$

Since

$$
0<\frac{2\left(1-|z|^{2}\right)}{\log \alpha-\log \left(1-|z|^{2}\right)} \leq \frac{1}{\log \alpha}
$$

and $\lim _{|z| \rightarrow 1} 1 /\left(\log \alpha-\log \left(1-|z|^{2}\right)\right)=0$, for $c / d>(2 \log \alpha)^{-1}$, the desired result follows by the almost maximum principle.

\section{Acknowledgements}

This work is part of the author's Ph.D. dissertation at POSTECH. She would like to express her gratitude to her advisor Professor Kang-Tae Kim for his guidance. 


\section{References}

[Bland 1986] J. S. Bland, "The Einstein-Kähler metric on $\left\{|\mathbf{z}|^{2}+|w|^{2 p}<1\right\}$ ", Michigan Math. J. 33:2 (1986), 209-220. MR 87i:32036 Zbl 0602.32005

[Fridman 1978] B. L. Fridman, "A class of analytic polyhedra", Dokl. Akad. Nauk SSSR 242:5 (1978), 1020-1022. In Russian; translated in Soviet Math. Dokl. 19:5 (1979), 1258-1261. MR 80b:32014 Zbl 0427.32013

[Greene and $\mathrm{Wu}$ 1971] R. E. Greene and H. Wu, "Curvature and complex analysis", Bull. Amer. Math. Soc. 77 (1971), 1045-1049. MR 44 \#473 Zbl 0225.32010

[Greene and $\mathrm{Wu} 1979] \mathrm{R}$. E. Greene and $\mathrm{H}$. Wu, Function theory on manifolds which possess a pole, Lecture Notes in Mathematics 699, Springer, Berlin, 1979. MR 81a:53002 Zbl 0414.53043

[Kim 1992] K.-T. Kim, "Domains in $\mathbf{C}^{n}$ with a piecewise Levi flat boundary which possess a noncompact automorphism group", Math. Ann. 292 (1992), 575-586. MR 93h:32024 Zbl 0735.32021

[Omori 1967] H. Omori, "Isometric immersions of Riemannian manifolds", J. Math. Soc. Japan 19 (1967), 205-214. MR 35 \#6101 Zbl 0154.21501

[Seshadri and Zheng 2008] H. Seshadri and F. Zheng, "Complex product manifolds cannot be negatively curved”, Asian J. Math. 12:1 (2008), 145-149. MR 2009d:53047 Zbl 1147.53317

[Yang 1976] P. C. Yang, "On Kähler manifolds with negative holomorphic bisectional curvature", Duke Math. J. 43:4 (1976), 871-874. MR 54 \#7837 Zbl 0358.32005

[Yau 1975] S. T. Yau, "Harmonic functions on complete Riemannian manifolds", Comm. Pure Appl. Math. 28 (1975), 201-228. MR 55 \#4042 Zbl 0291.31002

[Yau 1978] S. T. Yau, "A general Schwarz lemma for Kähler manifolds", Amer. J. Math. 100:1 (1978), 197-203. MR 58 \#6370 Zbl 0424.53040

[Yau 1982] S. T. Yau (editor), Seminar on Differential Geometry, Annals of Mathematics Studies 102, Princeton Univ. Press, 1982. MR 83a:53002

Received September 17, 2011. Revised December 5, 2011.

AERYEONG SEO

DEPARTMENT OF MATHEMATICS

Pohang University of Science And TeChNology (POSTECH)

POHANG 790-784

SOUTH KOREA

inno827@ postech.ac.kr 


\title{
PACIFIC JOURNAL OF MATHEMATICS
}

\author{
http://pacificmath.org \\ Founded in 1951 by \\ E. F. Beckenbach (1906-1982) and F. Wolf (1904-1989)
}

\section{EDITORS}

V. S. Varadarajan (Managing Editor)

Department of Mathematics

University of California

Los Angeles, CA 90095-1555

pacific@math.ucla.edu

Vyjayanthi Chari

Department of Mathematics

University of California

Riverside, CA 92521-0135

chari@math.ucr.edu

\section{Robert Finn}

Department of Mathematics Stanford University

Stanford, CA 94305-2125

finn@math.stanford.edu

Kefeng Liu

Department of Mathematics

University of California

Los Angeles, CA 90095-1555

liu@math.ucla.edu
Darren Long

Department of Mathematics

University of California

Santa Barbara, CA 93106-3080

long@math.ucsb.edu

Jiang-Hua Lu

Department of Mathematics

The University of Hong Kong

Pokfulam Rd., Hong Kong jhlu@maths.hku.hk

Alexander Merkurjev

Department of Mathematics

University of California

Los Angeles, CA 90095-1555

merkurev@math.ucla.edu
Sorin Popa

Department of Mathematics University of California

Los Angeles, CA 90095-1555 popa@math.ucla.edu

Jie Qing

Department of Mathematics

University of California

Santa Cruz, CA 95064

qing@cats.ucsc.edu

Jonathan Rogawski

Department of Mathematics

University of California

Los Angeles, CA 90095-1555

jonr@math.ucla.edu

\section{PRODUCTION}

pacific@math.berkeley.edu

\section{SUPPORTING INSTITUTIONS}

ACADEMIA SINICA, TAIPEI

CALIFORNIA INST. OF TECHNOLOGY INST. DE MATEMÁTICA PURA E APLICADA KEIO UNIVERSITY

MATH. SCIENCES RESEARCH INSTITUTE NEW MEXICO STATE UNIV.

OREGON STATE UNIV.

\author{
STANFORD UNIVERSITY \\ UNIV. OF BRITISH COLUMBIA \\ UNIV. OF CALIFORNIA, BERKELEY \\ UNIV. OF CALIFORNIA, DAVIS \\ UNIV. OF CALIFORNIA, LOS ANGELES \\ UNIV. OF CALIFORNIA, RIVERSIDE \\ UNIV. OF CALIFORNIA, SAN DIEGO \\ UNIV. OF CALIF., SANTA BARBARA
}

\author{
UNIV. OF CALIF., SANTA CRUZ \\ UNIV. OF MONTANA \\ UNIV. OF OREGON \\ UNIV. OF SOUTHERN CALIFORNIA \\ UNIV. OF UTAH \\ UNIV. OF WASHINGTON \\ WASHINGTON STATE UNIVERSITY
}

These supporting institutions contribute to the cost of publication of this Journal, but they are not owners or publishers and have no responsibility for its contents or policies.

See inside back cover or pacificmath.org for submission instructions.

The subscription price for 2012 is US \$420/year for the electronic version, and \$485/year for print and electronic.

Subscriptions, requests for back issues from the last three years and changes of subscribers address should be sent to Pacific Journal of Mathematics, P.O. Box 4163, Berkeley, CA 94704-0163, U.S.A. Prior back issues are obtainable from Periodicals Service Company, 11 Main Street, Germantown, NY 12526-5635. The Pacific Journal of Mathematics is indexed by Mathematical Reviews, Zentralblatt MATH, PASCAL CNRS Index, Referativnyi Zhurnal, Current Mathematical Publications and the Science Citation Index.

The Pacific Journal of Mathematics (ISSN 0030-8730) at the University of California, c/o Department of Mathematics, 969 Evans Hall, Berkeley, CA 94720-3840, is published monthly except July and August. Periodical rate postage paid at Berkeley, CA 94704, and additional mailing offices. POSTMASTER: send address changes to Pacific Journal of Mathematics, P.O. Box 4163, Berkeley, CA 94704-0163.

PJM peer review and production are managed by EditFLOW ${ }^{\mathrm{TM}}$ from Mathematical Sciences Publishers.

PUBLISHED BY PACIFIC JOURNAL OF MATHEMATICS

at the University of California, Berkeley 94720-3840

A NON-PROFIT CORPORATION

Typeset in LATEX

Copyright $(02012$ by Pacific Journal of Mathematics 


\section{PACIFIC JOURNAL OF MATHEMATICS}

Volume $256 \quad$ No. $1 \quad$ March 2012

On slim double Lie groupoids

NiCOlas ANDruskiewitsch, JEsus OCHOA ARANGO and AlEJANDRO

TIRABOSCHI

Topological classification of quasitoric manifolds with second Betti number 2

19

Suyoung Choi, SeOnjeOng PARK and Dong Youp SuH

Refined Kato inequalities for harmonic fields on Kähler manifolds

DANiEL Cibotaru and PENG ZHU

Deformation retracts to the fat diagonal and applications to the existence of peak solutions of nonlinear elliptic equations

E. Norman Dancer, Jonathan Hillman and Angela Pistoia

Descent for differential Galois theory of difference equations: confluence and $q$-dependence

LuCia Di Vizio and Charlotte HaRdouin

Modulation and natural valued quiver of an algebra

FANG LI

Willmore hypersurfaces with two distinct principal curvatures in $\mathbb{R}^{n+1}$

TONGZHU LI

Variational inequality for conditional pressure on a Borel subset

YuAN Li, ERCAI CHEN and WEN-ChIAO CHENG

New homotopy 4-spheres

DANIEL NASH

Combinatorial constructions of three-dimensional small covers

YASUZO NISHIMURA

On a theorem of Paul Yang on negatively pinched bisectional curvature

AERYEONG SEO

Orders of elements in finite quotients of Kleinian groups

PETER B. Shalen

A new algorithm for finding an 1.c.r. set in certain two-sided cells

JIAN-YI SHI

Addendum to the article Superconnections and parallel transport

FLORIN DUMITRESCU 